\title{
Ras signaling contributes to survival of human T-cell leukemia/ lymphoma virus type 1 (HTLV-1) Tax-positive T-cells
}

\author{
Giovanna Stoppa $\cdot$ Enrica Rumiato • \\ Daniela Saggioro
}

Published online: 30 November 2011

(c) The Author(s) 2011. This article is published with open access at Springerlink.com

\begin{abstract}
Ras signaling pathways play an important role in cellular proliferation and survival, and inappropriate activation of Ras frequently results in cell transformation and cancer. Human T-cell leukemia/lymphoma virus type 1 (HTLV-1) is the etiological agent of the adult T-cell leukemia/lymphoma (ATLL), a severe malignancy that has a poor prognosis and exhibits resistance to conventional chemotherapy. Although the mechanisms involved in cell transformation by HTLV-1 have not been completely clarified, it is generally thought that Tax plays a pivotal role in the process. We have previously proposed that a functionally active Ras protein is needed for efficient antiapoptotic activity of Tax. In this study we report data indicating that the apoptotic resistance of cells expressing Tax, constitutively or transiently, is linked to the intracellular levels of Ras-GTP. Indeed, we found that Tax-positive cells have a high content of active Ras, and that inhibition of Ras signaling, using the antagonist farnesyl thyosalicylic acid (FTS), increases their sensitivity to apoptosis. FTS treatment was also accompanied by a decrease in ERK, but not Akt, phosphorylation. Thus, all together our data suggest that the interaction between Tax and Ras could be important to ATLL pathogenesis, and indicate Ras as a possible target for therapeutic intervention in ATLL patients.
\end{abstract}

Keywords HTLV-1 - Tax - Ras - Apoptosis - ERK1/2 . Akt

G. Stoppa $\cdot$ E. Rumiato $\cdot$ D. Saggioro $(\varangle)$

Immunology and Molecular Oncology Unit, Veneto Institute of Oncology IOV_-IRCCS, via Gattamelata 64, 35128 Padua, Italy

e-mail: d.saggioro@unipd.it; daniela.saggioro@ioveneto.it

\section{Introduction}

Ras proteins are small GTPases that function as molecular switches, alternating between inactive (GDP-bound) and active (GTP-bound) states. Like many genes involved in the regulation of multiple cellular signaling pathways (i.e., differentiation, proliferation and survival), Ras when aberrantly expressed, contributes to cancer development [1-4]. We have previously described a possible link between Ras signaling and resistance to apoptosis of cells expressing the Tax protein of Human T-cell leukemia virus type $1(\mathrm{HTLV}-1)$ [5].

Human T-cell leukemia virus type 1 is the causative agent of adult T-cell leukemia/lymphoma (ATLL) $[6,7]$ and HTLV-1 associated myelopathy/tropical spastic paraparesis (HAM/TSP) [8]. Even though the mechanisms by which the virus engenders disease are not yet completely understood, numerous evidences indicate the multifunctional Tax protein as essential for malignant transformation (reviewed in [9]). Indeed, Tax causes leukemia in transgenic mice [10], and immortalizes human lymphocytes when expressed in either a herpes- or retroviral vector $[11,12]$. This oncogenic potential is accounted for by Tax ability to modulate the synthesis or the activity of many cellular proteins that control a variety of fundamental cellular processes [13].

A key feature of malignant transformation is the induction of apoptotic resistance [14], and aberrant cell death is usually associated with uncontrolled cell growth. Tax contribution to apoptosis is still controversial since the protein was shown to possess both anti-apoptotic and proapoptotic activity [15-17]. However, at present, it is generally accepted that the anti-apoptotic activity of Tax overrides its potential pro-apoptotic effects.

In previous studies, using murine fibroblasts and human HeLa cells, we have shown that Tax expression induces 
resistance to apoptosis triggered by different stimuli. Analysis of potential mechanisms revealed that the observed resistance was linked to high levels of transcriptionally active CREB and to the presence of a functional Ras protein [5, 18, 19].

In this study, carried out on human T-cells, we report data indicating that Tax-expressing cells have high levels of Ras-GTP (active form), and we demonstrate that RasGTP contributes to Tax-mediated apoptosis protection. Indeed, treatment of cells with a Ras antagonist decreased their apoptotic resistance; treatment was also accompanied by a reduction of both Ras-GTP and phosphorylated ERK1/2.

All together, our results suggest an important role for Ras signaling in Tax-mediated apoptotic resistance and support the notion that Ras activation might be important for ATLL development.

\section{Materials and methods}

Cell culture and plasmid

Jurkat, and derived clones, Hut-102, JPX9, and Molt3 cell lines were maintained in RPMI 1640 medium supplemented with $10 \%$ fetal bovine serum (FBS) and $2 \mathrm{mM}$ L-glutamine. HeLa-tat cells were cultured in Dulbecco's modified Eagle's medium (DMEM) supplemented with $10 \%$ FBS and $2 \mathrm{mM}$ L-glutamine. Jurkat clones expressing Tax were obtained by electroporation with the Taxexpressing plasmid pLcXL [20], together with a plasmid expressing the neomycin-resistance gene (p-exo-neo). Following selection with G418, the resulting clones were expanded and characterized. In parallel, Jurkat cells were also electroporated with the p-exo-neo vector alone to generate control clones. Fugene 6 (Roche Diagnostics, Mannheim, Germany) was used to transiently transfect HeLa-tat cells with a plasmid expressing Tax under the control of the cytomegalovirus promoter (pCMV-Tax) [21]. Expression of Tax in JPX9 cells was induced by addition of $\mathrm{CdCl}_{2}$ to a final concentration of $10 \mu \mathrm{M}$.

Western blot assay and Ras-GTP selective precipitation

Total proteins were extracted directly in Laemmli buffer; a cocktail of protease inhibitors (Roche) was included in every extraction. Equal volumes of cell lysates, corresponding to $4 \times 10^{5}$ Jurkat cells or $5 \times 10^{4} \mathrm{HeLa}$ cells (or $30 \mu \mathrm{g}$ of proteins quantified using the Coomassie Plus Reagent kit, Thermo Scientific) were separated by SDSPAGE electrophoresis in $10 \%$ or $15 \%$ gels and then transferred to PVDF membranes (Amersham GE Healthcare, UK). Cytosolic fractions were prepared by selective plasma membrane permeabilization with $0.05 \%$ digitonin in an isotonic sucrose buffer, as previously described [19]. RasGTP was identified by precipitation with Gst-tagged Raf1RBD (Ras Activation Assay Kit; Cytoskeleton Inc., Denver, CO, USA) followed by immunoblotting. The following antibodies were used: rabbit anti-Tax serum obtained from the AIDS Research Reagents Collection [22], mouse monoclonal anti-pan-Ras (Sigma-Aldrich Inc., St Luis, MO, USA or Cytoskeleton Inc.), monoclonal antibody $7 \mathrm{H} 8.2 \mathrm{C} 12$ against cytochrome $c$ (BD Biosciences, Franklin Lakes, NJ, USA), goat anti-Hsp60 polyclonal antibody N-20 (Santa Cruz Biotechnology), mouse monoclonal anti-tubulin (Sigma-Aldrich Inc.), rabbit polyclonal anti-PARP, rabbit polyclonal anti-cleaved caspase 3, mouse monoclonal antiphosphorylated Ser-473 Akt, rabbit polyclonal anti-total Akt, rabbit polyclonal anti-total ERK1/2, and mouse monoclonal anti-phosphorylared ERK1/2, all from Cell Signaling Technology (Danvers, MA, USA).

Annexin V cell death assay

Jurkat and JD1 cells $\left(2 \times 10^{5} / \mathrm{ml}\right)$ were treated for $24 \mathrm{~h}$ with $10 \mu \mathrm{M}$ cisplatin. The Annexin $\mathrm{V}$ assay was performed using an Annexin V-FITC (Roche), according to manufacturer's instructions. In brief, $5 \times 10^{5}$ cells were collected, centrifuged, rinsed with Annexin-binding buffer (RPMI containing $10 \mathrm{mM}$ HEPES and $0.1 \%$ bovine serum albumine (BSA)) and then incubated in $500 \mu \mathrm{l}$ of RPMI/ HEPES/BSA buffer containing $1 \mu \mathrm{g} / \mathrm{ml}$ propidium iodide and $10 \mu \mathrm{l} / \mathrm{ml}$ Annexin V-FITC for $10 \mathrm{~min}$ at room temperature. The samples were analyzed by flow cytometry using a Coulter Epicsxl, with 10,000 ungated events examined for each sample.

\section{Confocal microscopy analysis}

HeLa-tat cells were seeded on glass coverslips in $35 \mathrm{~mm}$ tissue culture plates at a concentration of $2 \times 10^{5}$ cells. Twenty-four hours after transfection, cells were treated with $60 \mu \mathrm{M}$ cisplatin for $4-6 \mathrm{~h}$ and than fixed for $30 \mathrm{~min}$ in $4 \%$ formaldehyde, permeabilized for 2 min with $0.2 \%$ Triton X-100/PBS and immunodecorated. The murine anticytochrome $c$ monoclonal antibody 6H2.B4 (BD Biosciences) and the rabbit anti-Tax serum (see above) were used as primary antibodies. Alexa 488- and Alexa 546-conjugated secondary antibodies were purchased from Molecular Probes. Coverslips were analyzed using a Zeiss LSM510 confocal laser microscope.

Chloramphenicol acetyl transferase (CAT) assay

Parental Jurkat cells and derived clones were transiently transfected by the DEAE-Dextran method [23] with the 
CAT reporter construct LTR-CAT (pU3R1) [23] together with a CMV- $\beta$-gal plasmid. Twenty-four hours later the cells were collected, rinsed with PBS and lysed by three freeze-thaw cycles in $50 \mu \mathrm{l}$ of $0.25 \mathrm{M}$ Tris-HCl $\mathrm{pH} 7.8$. Aliquots of the supernatants were assayed for CAT activity as previously described [24]; the percentage of chloramphenicol conversion to its acetylated forms was determined using an InstantImmager (Packard Instrument Company, Meriden, CT, USA), and normalized for $\beta$-Gal activity.

\section{Results}

Generation and characterization of Tax-expressing T-cells

To assess the role of Tax protein in the apoptotic behavior of T-cells, we generated stable Tax-positive T-cells by transfecting the Jurkat cell line with a plasmid expressing Tax under the control of the HTLV-1 LTR, together with a plasmid expressing neomycin-resistance. Transfected cells were, thus, selected with neomycin and the resulting clones expanded. Corresponding controls were generated by transfecting Jurkat cells with mock DNA together with the selectable plasmid. The stable expression of Tax was verified by both CAT assay and Western blot. Results showed that only one of the isolated clones, named JD1, expressed an active and detectable Tax protein (Fig. 1a, b), suggesting that in the other clones tax was, most likely, lost or inactivated.

When we tested the resistance of the isolated clones to cisplatin-induced apoptosis, we found that only JD1 cells were resistant to death, measured as percentage of PARP cleavage, after $24 \mathrm{~h}$ treatment. In contrast, the parental Jurkat cells, the mock-transfected clones as well as the Tax-transfected cells that had lost Tax activity, were susceptible to cisplatin treatment (Fig. 1c). The resistance of JD1 cells to apoptosis induced by cisplatin treatment was also confirmed by Annexin V cell death assay (Fig. 1d). However, by changing the duration of treatment to $48 \mathrm{~h}$ (Fig. 1e) or the drug dosage (data not shown), it was possible to increase the percentage of apoptotic JD1 cells. Similar results were obtained when the cells, after $24 \mathrm{~h}$ of treatment were grown for additional $24 \mathrm{~h}$ in drug free medium. In this experimental setting, both Jurkat and JD1 cells resulted susceptible to drug doses that were ineffective, or only slightly effective, after $24 \mathrm{~h}$ (Fig. 1f). These results suggested that the apoptotic machinery was still working in JD1 cells and that their resistance was probably due to an increased threshold of sensitivity to apoptotic stimuli. JD1 cells exhibited similar behavior when other apoptosis inducers, such as etoposide or ceramide, were used (not shown).
It has been reported that Tax can transactivate the MDR1 (multidrug resistance) gene promoter [25] and that ATLL cells very often show an enhanced MDR-1 expression [26]. Hence, we tested whether the observed increased resistance of JD1 cells could be ascribable to P-glycoprotein overexpression. As reported in Fig. 1g, we found that concomitant treatment with cisplatin and verapamil, a known inhibitor of the MDR pump [27], induced an increase in the death percentage of JD1 cells; however, a similar increment was also observed in Jurkat cells indicating that JD1 and Jurkat cells have comparable MDR-1 pump activity and that other mechanisms of resistance should be at work in JD1 cells (Fig. 1g).

Resistance to apoptosis is not restricted to JD1 cells

To determine whether resistance to apoptosis was restricted to JD1 cells or was instead a more general phenomenon in Tax-positive T-cells, we analyzed the response to cisplatin treatment of Hut-102 and JPX9 cell lines. Hut-102 is an ATLL derived cell line expressing constitutively a high amount of Tax [28]. On the contrary, JPX9 is a Taxinducible cell line generated by introducing in Jurkat cells the Tax gene under the control of a metallothionein promoter [29], and expression of Tax is induced by cells treatment with heavy metals. As reported in Fig. 2a, Hut102 cells exhibited a higher resistance to cisplatin-induced apoptosis than the non-infected MOLT3 T-cells, included as control. Comparable results were obtained with JPX9 cells, since the induction of Tax expression by $\mathrm{CdCl}_{2}$ treatment was accompanied by a decreased sensitivity to cisplatin (Fig. 2b).

Tax expression blocks cytochrome $c$ release from mitochondrial intermembrane compartment

We previously reported that Tax induces resistance to apoptotic stimuli, such as growth factors withdrawal or Bax overexpression, by interfering with the mitochondrial apoptotic pathway $[18,19,30]$. As mitochondria play a pivotal role in the control of apoptosis triggered by chemotherapeutic drugs, we investigated whether Tax interferes with cisplatin-induced apoptosis by inhibiting the so called intrinsic apoptotic pathway. Thus, as a parameter of mitochondrial engagement, we tested the release of cytochrome $c$ from mitochondria, a key event in the mitochondrial apoptotic pathway, after cisplatin treatment. We found that in a culture of HeLa-tat cells transiently transfected with a Tax-expressing plasmid and then treated with cisplatin, cytochrome $c$ release was present almost exclusively in the Tax-negative cells (Fig. 3a, b). Similarly, after extraction with digitonin, that selectively permeabilizes plasma and outer mitochondrial membranes to derive 

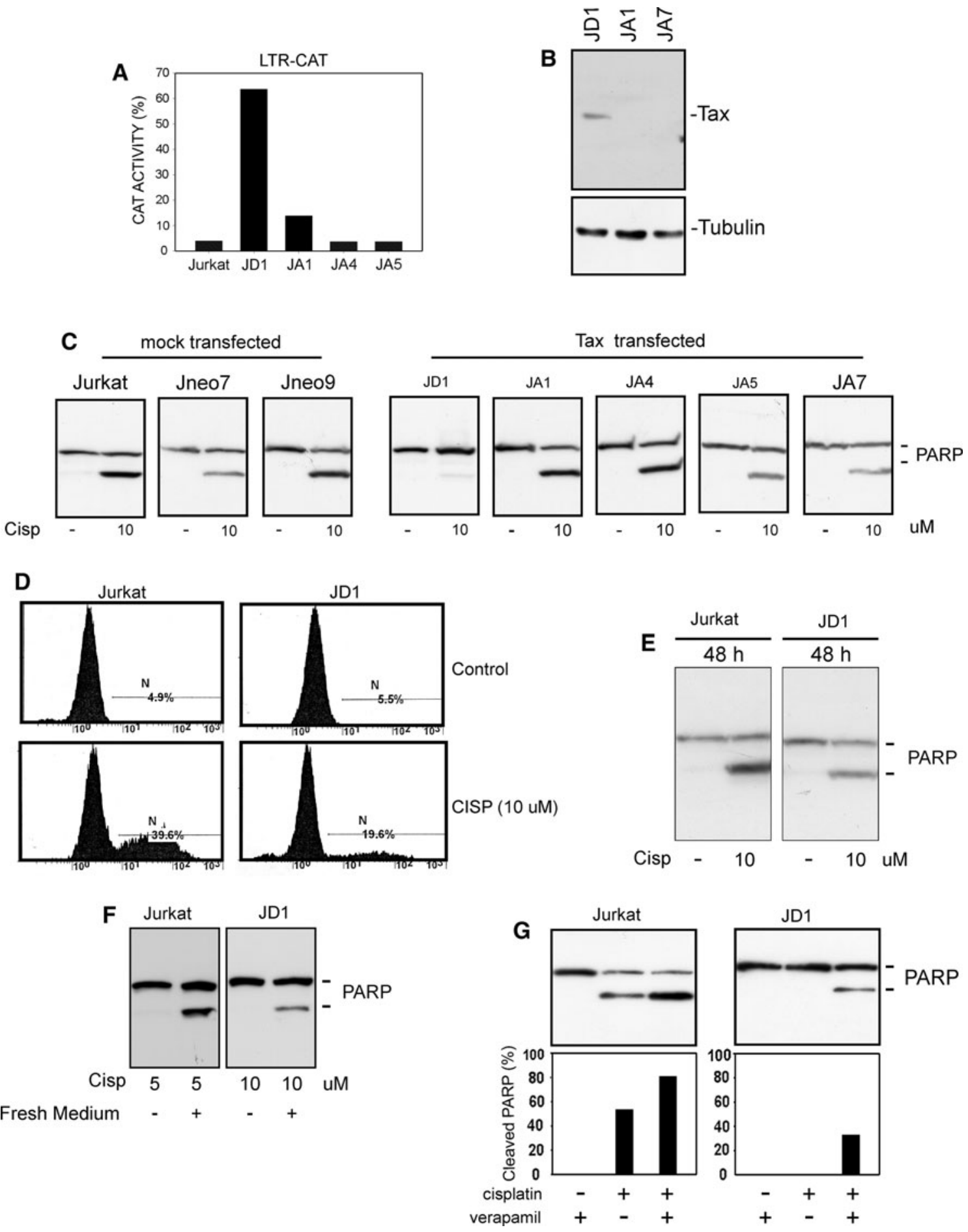

cytosolic protein fraction [31], no cytochrome $c$ could be visualized in the cytosolic fraction of JD1 cells (Fig. 3c). These results indicate that Tax protects T-cells from cisplatin-induced apoptosis by blocking the mitochondrial apoptotic pathway. In line with these findings, no activation of the caspase 3 was observed in JD1 cells after treatment with cisplatin (Fig. 3c).
Ras activation is responsible for the apoptotic resistance of Tax-expressing cells

We then sought to investigate the molecular mechanisms that could make Tax-expressing cells more resistant to apoptotic stimuli that trigger the mitochondrial pathway. Our previous data, in HeLa cell system, suggested the participation of Ras 
4 Fig. 1 Phenotypic characterization of Jurkat-derived clones. a Analysis of Tax activity in Tax-transfected Jurkat derivatives. Cells were transiently transfected with an LTR-CAT plasmid; the $\beta$-galactosidase expression vector CMV- $\beta$-gal was added as a control of transfection efficiency. Percentage of chloramphenicol acetylation was calculated as described in "Materials and methods" and normalized for $\beta$-gal activity. b Western blot analysis of Tax expression in Jurkat cell derivatives; tubulin is shown as a loading control. c Western blot analysis of PARP cleavage after treatment with cisplatin for $24 \mathrm{~h}$. Aliquots corresponding to $4 \times 10^{5}$ cells (or to $30 \mu \mathrm{g}$ of total proteins) were subjected to SDS-PAGE, transferred to PVDF membranes and then immunodecorated with an anti-PARP antibody. d Annexin V cell death assay in Jurkat and JD1 cells treated with cisplatin for $24 \mathrm{~h}$. e Western blot analysis of PARP cleavage in Jurkat and JD1 cells treated for $48 \mathrm{~h}$ with cisplatin. f Western blot analysis of cell death susceptibility of Jurkat and JD1 cells treated with cisplatin for $24 \mathrm{~h}$ and then growth for additional $24 \mathrm{~h}$ in cisplatin-free medium. $\mathrm{g}$ Analysis of the effect of cisplatin treatment in the presence of the MDR1 inhibitor verapamil. Cells were treated with cisplatin for $24 \mathrm{~h}$ in the presence of $20 \mu \mathrm{g} / \mathrm{ml}$ verapamil. At the end of treatment proteins were extracted as described in the "Materials and methods" and analyzed by Western blot. Histograms represent percentage of cleaved PARP, resulting from densitometric analysis

signaling in Tax-mediated protection from apoptosis. Indeed, we showed that Tax interacts with the GTPase activating protein $\mathrm{GAP}^{1 \mathrm{~m}}$. As GAPs favor the hydrolysis of Ras-GTP (active form) into Ras-GDP (inactive form), our working model proposed the presence of high levels of activated Ras in Tax-expressing cells due to a Tax-mediated inefficient conversion of Ras-GTP into Ras-GDP [5].

Here, to substantiate this hypothesis, we compared the amount of Ras-GTP present in Tax-positive JD1 cells with that of parental Jurkat cells and apoptosis-sensitive Jurkatderived clones. The analysis was carried out by exploiting the greater affinity of Raf1-RBD (Ras-binding domain) for Ras-GTP. Indeed, Raf1-RBD binds very tightly to the GTP-bound form of Ras, while RBD affinity for Ras-GDP is three orders of magnitude lower [32]. As reported in Fig. 4, we found that JD1 cells have levels of Ras-GTP higher than those found in parental Jurkat cells and in Taxnegative Jneo3 and Jneo6 clones (Fig. 4a). Interestingly, the JA1 clone, derived from the same transfection as the JD1 cells, but that had lost Tax activity (Fig. 1a) and was cisplatin-susceptible (Fig. 1c), also showed a low level of Ras-GTP (Fig. 4a). Consistently, an increased amount of activated Ras was also detected in the Tax-inducible JPX9 cells after treatment with $\mathrm{CdCl}_{2}$ (Fig. 4b) and in Taxtransfected HeLa-tat cells (Fig. 4c). No induction of Ras activation was observed in mock-transfected HeLa-tat cells, and more interestingly in the same cells transfected with the non-protective Tax-mutant M47 (Fig. 4c).

These findings strongly indicate that the activation of Ras signaling could be one of the mechanisms by which Tax increases resistance to cisplatin-induced cell death.

Ras controls several effector proteins, including PI3K/ Akt and MEK/ERK, that regulate signaling involved in cell
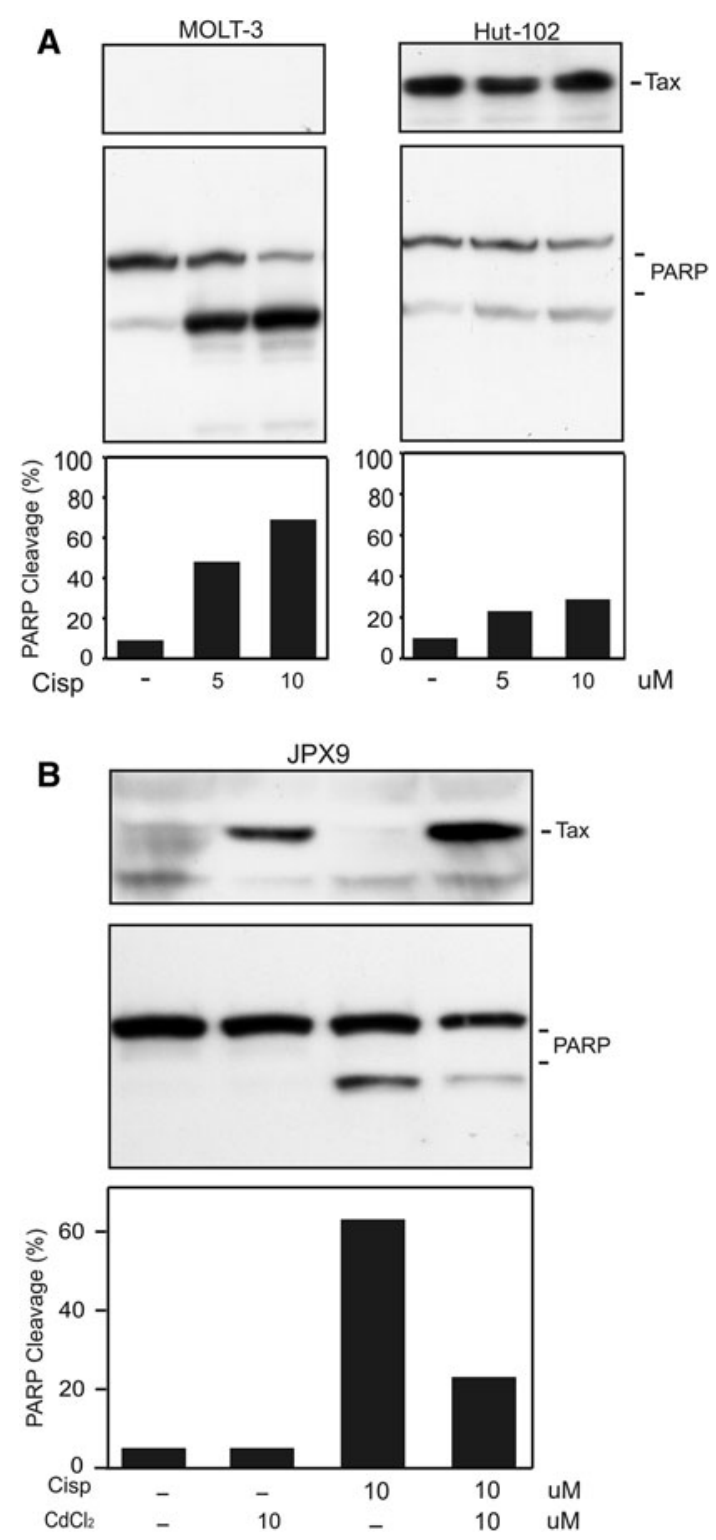

Fig. 2 Effect of cisplatin treatment in HTLV-1 infected Hut-102 or Tax-inducible JPX9 cell lines. PARP cleavage after cisplatin treatment was evaluated by Western blot. Protein aliquots corresponding to $4 \times 10^{5}$ of HTLV-1 infected Hut-102 and HTLV-1 negative control MOLT-3 (a) or Tax-inducible JPX9 (b) cells were separated by SDS-PAGE, transferred to PVDF membranes and then immunodecorated with anti-PARP or anti-Tax antibodies. To induce Tax expression, JPX9 cells were grown for $24 \mathrm{~h}$ in medium containing $10 \mu \mathrm{M} \mathrm{CdCl}_{2}$ before treatment with cisplatin. Histograms represent percentage of cleaved PARP, resulting from densitometric analysis

survival and CREB phosphorylation. Akt has been reported to be activated in HTLV-1 transformed cells or Taxexpressing cells [33-35] and we showed that the MEK/ ERK pathway is involved in Tax-mediated apoptosis protection, in HeLa cells [5]. Hence, we compared the phosphorylation state of Akt and ERK proteins in JD1 and parental Jurkat cells. As reported in Fig. 4d, JD1 cells 

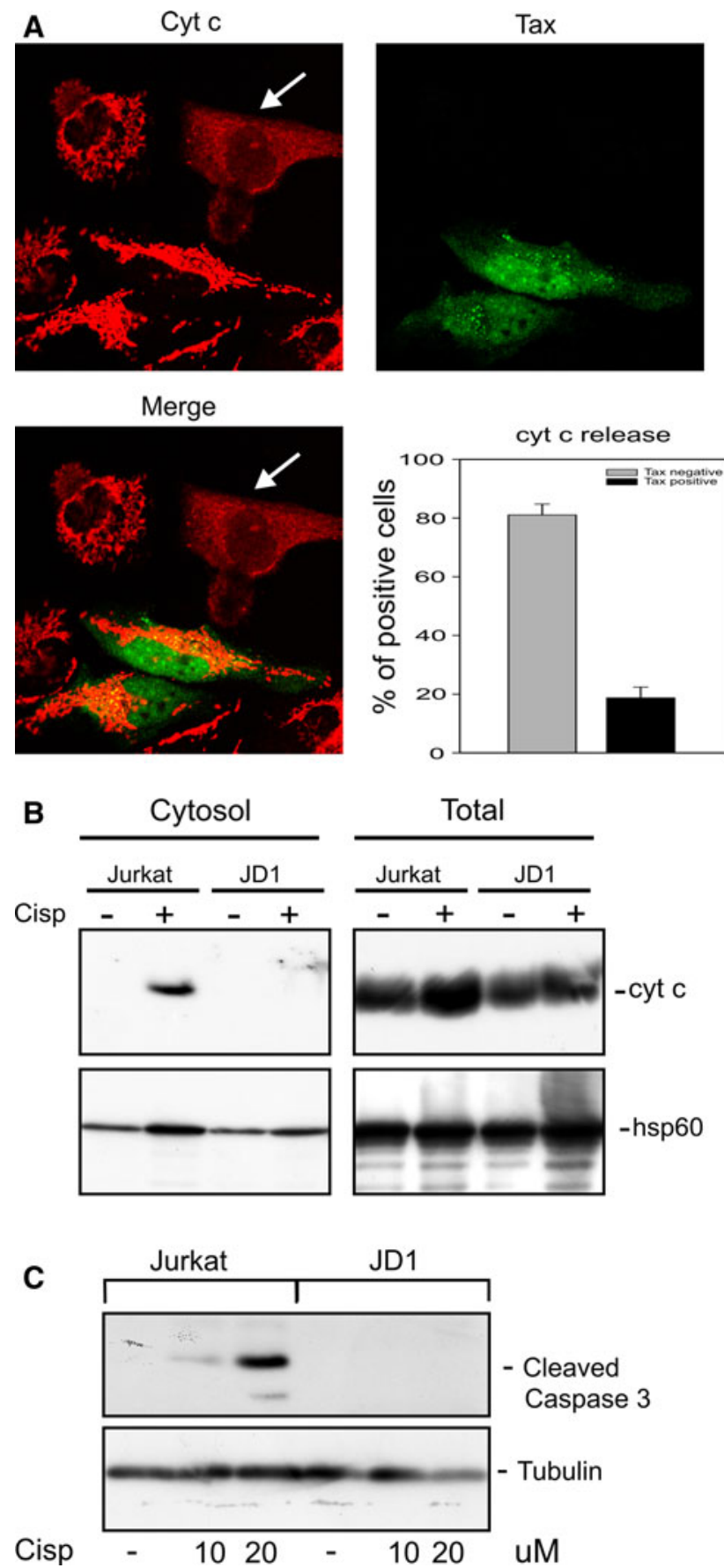

exhibited a much higher amount of phosphorylated Akt and ERK than Jurkat cells, likely as a consequence of the increased amount of activated Ras.

Inhibition of Ras signaling reduces resistance to apoptosis of Tax-positive cells

To verify whether increased Ras activation is involved in Tax-mediated apoptotic resistance and enhanced Akt and
4 Fig. 3 Analysis of cytochrome $c$ distribution in Tax-positive and Tax-negative cell lines after cisplatin treatment. a Confocal analysis of HeLa-tat cells transiently transfected with a CMV-Tax plasmid and then treated with $60 \mu \mathrm{M}$ cisplatin for $6 \mathrm{~h}$. Arrows indicate cells with cytochrome $c$ release. Graph represents percentage of Tax-positive and Tax-negative HeLa-tat cells exhibiting cytochrome $c$ release; data report mean values \pm SE of at least 600 cells randomly counted in three independent experiments. b Western blot analysis of cytochrome $c$ release in Jurkat and JD1 cells after treatment with $5 \mu \mathrm{M}$ cisplatin for $24 \mathrm{~h}$. Digitonin-extracted cytosolic or total protein fractions were separated by SDS-PAGE and immunodecorated using antibody specific for cytochrome $c$. The hsp60 antibody was used as control. c Western blot analysis of caspase 3 activation in Jurkat and JD1 cells after treatment with cisplatin for $24 \mathrm{~h}$; tubulin is shown as a loading control

ERK phosphorylation, we treated the cells with farnesyl thyosalicylic acid (FTS). FTS is a Ras farnesylcystein mimetic, that selectively disrupts the association of active Ras protein with the plasma membrane and can thus be considered as a Ras inhibitor [36].

At first we evaluated the effect of FTS treatment on RasGTP levels. As reported in Fig. 5a, when JD1 cells were treated for $24 \mathrm{~h}$ with a not toxic dose of FTS $(50 \mu \mathrm{M})$, we observed a considerable reduction in the active form of Ras compared to untreated control cells. Analysis of the apoptotic behavior of JD1 cells treated with the Ras inhibitor, indicated that the combined administration of cisplatin and FTS increased the cytotoxic effects of cisplatin in JD1 cells (Fig. 5b); similar results were obtained when different timing of treatment and different doses of cisplatin were used (Fig. 5c).

Since, as reported in Fig. 4c, JD1 cells have high levels of activated Akt and ERK proteins, we analyzed these proteins after FTS treatment. Western blot analysis showed that while the level of phospho-Akt did not change, the amount of phospho-ERK decreased considerably after treatment (Fig. 5d). All together, these results further support the hypothesis that an active Ras plays a relevant role on Tax-mediated resistance to apoptosis and implicate the MAPK/ERK pathway as one of its effectors.

\section{Discussion}

Ras family proteins cycle between inactive GDP-bound and active GTP-bound states and function as molecular switches for signaling pathways that regulate many aspects of cell behavior including cell division, differentiation, transformation and survival [2].

It has also been reported that Ras plays a relevant role in T-cell maturation and accumulates in the active GTPbound form in response to some cytokines or to T-cell antigen receptor (TCR) stimulation [37]. Deregulated Ras signaling is present in numerous human cancers, including 


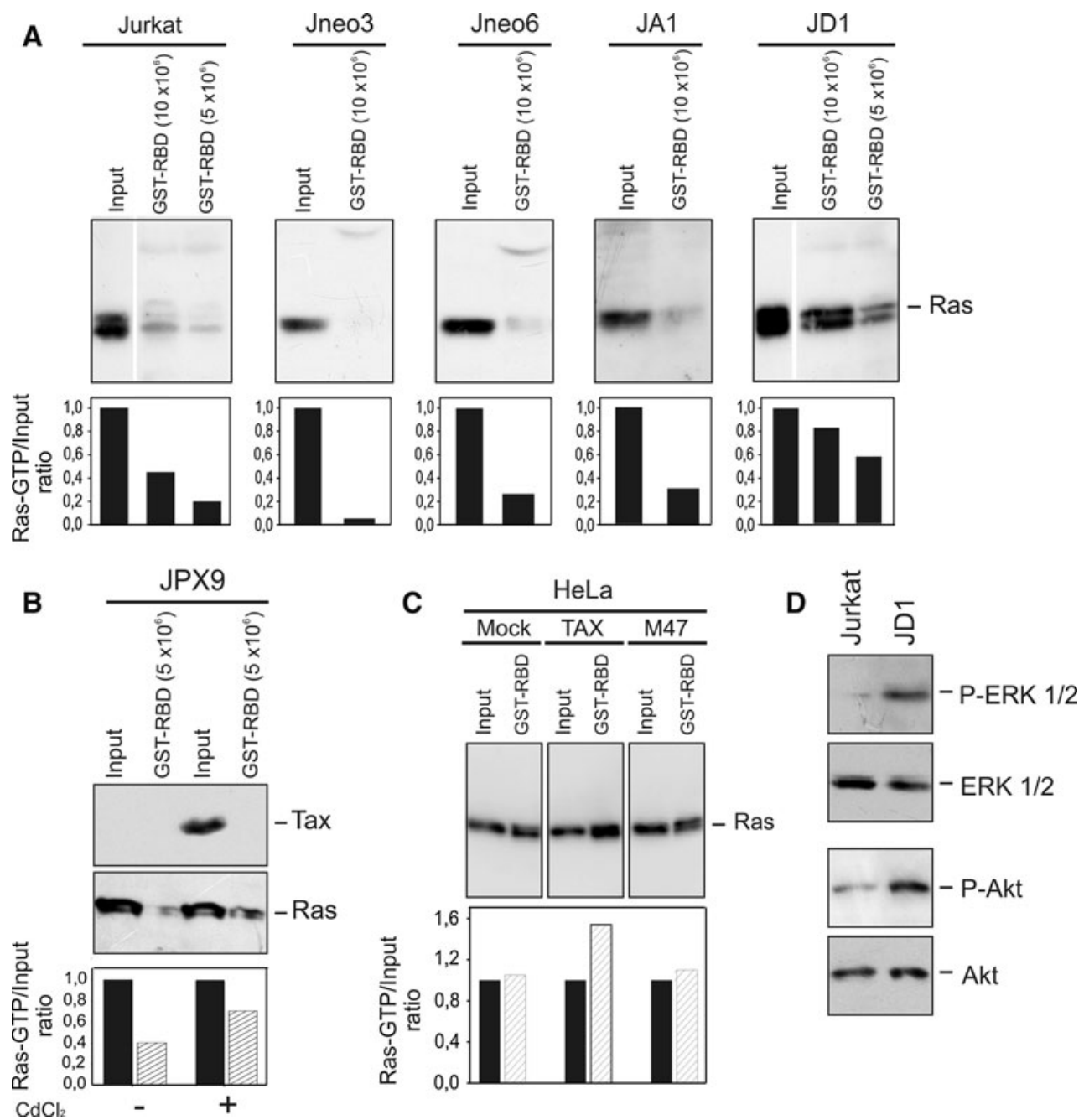

Fig. 4 Tax-expressing cells exhibit high levels of Ras-GTP, and ERK1/2 and Akt phosphorylation. Lysates from 10 or $5 \times 10^{6}$ Taxnegative (Jurkat, Jneo3, Jneo6 and JA1) or -positive (JD1) cells (a), and from $5 \times 10^{6}$ Tax-inducible JPX9 cells (b), were precipitated using Gst-tagged Raf1-RBD and subjected to Western blot. Precipitated RasGTP and total Ras (input) were detected using a pan-Ras antibody, as described in "Materials and methods"; input represents $1 / 60$ of total lysate used for precipitation. To induce Tax expression, JPX9 cells were grown $24 \mathrm{~h}$ before precipitation in medium containing $10 \mu \mathrm{M} \mathrm{CdCl} 2$. c Detection of Tax-induced Ras-GTP in

hematopoietic disorders [1, 38-40]. An active Ras might contribute to both inhibition of apoptosis, and development of tumor cell resistance to chemotherapy and ionizing radiation [41, 42]. Deregulated Ras activation is achieved mainly through mutations [1]; however, malfunction of GTPase activating proteins (GAPs), that regulate the conversion of GTP-bound to GDP-bound Ras forms, can also affect the intracellular level of activated Ras [43].

HTLV-1 is a deltaretrovirus that immortalizes and transforms human $\mathrm{T}$ lymphocytes and is the causative agent of the ATLL; prognosis for ATLL patients remains poor because of tumor cells resistance to conventional,
HeLa-tat after transient transfection with plasmids expressing Tax wild type or its mutant M47. Cells lysate was precipitated with Gsttagged Raf1-RBD followed by blotting and immunodecoration with a pan-Ras antibody. Histograms in $\mathbf{a}, \mathbf{b}$, and $\mathbf{c}$ represent the ratio between Ras-GTP and the input, resulting from densitometric analysis. d Analysis of phosphorylated forms of Akt and ERK in Jurkat and JD1 cells. Aliquots corresponding to $30 \mu \mathrm{g}$ of total proteins were subjected to Western blot and immunodecorated with antibodies specific for ERK1/2 and Akt or their phosphorylated (P) forms

apoptosis-inducing, chemotherapeutic agents. Although many HTLV-1 basic functions have been clarified, therapeutic strategies are still unsuccessful, with few responder patients and a poor survival [16]. Tax protein, with its pleiotropic activity, is considered the oncogenic product of HTLV-1 and the main culprit of cell death resistance in infected tumor cells.

We previously provided evidence that Tax needs a functionally active Ras protein for the induction of apoptotic resistance [5]. Furthermore, we and others have shown that Tax co-precipitates with the Ras-GAP ${ }^{1 \mathrm{~m}}$ protein [5, 44]. On the basis of these findings, we proposed a working 
Fig. 5 Treatment with the Ras inhibitor FTS acid sensitizes Tax-positive cells to cisplatininduced cell death. a Lysates of JD1 cells treated with FTS $(50 \mu \mathrm{M})$ for $24 \mathrm{~h}$ were precipitated using Gst-tagged Raf1-RBD and immunoblotted. Ras was detected with a panRas antibody. Histogram represents densitometric analysis of the Ras-GTP/input ratio. b Cells were treated with cisplatin and FTS $(50 \mu \mathrm{M})$ for $24 \mathrm{~h}$ and then processed for PARP analysis. Histograms represent ratio between cleaved and uncleaved PARP forms, resulting from densitometric analysis. c FTS $(50 \mu \mathrm{M})$ and cisplatin were added at the same time. After $24 \mathrm{~h}$ the culture medium was changed and cells were post incubated in cisplatinfree medium with or without FTS $(50 \mu \mathrm{M})$. Actin was included as loading control. Histograms represent ratio between cleaved and uncleaved PARP forms obtained by densitometric analysis. d Effect of FTS on the phosphorylated form of Akt and ERK in JD1 cells. Total proteins and phosphorylated $(\mathrm{P})$ forms were detected as in Fig. 4

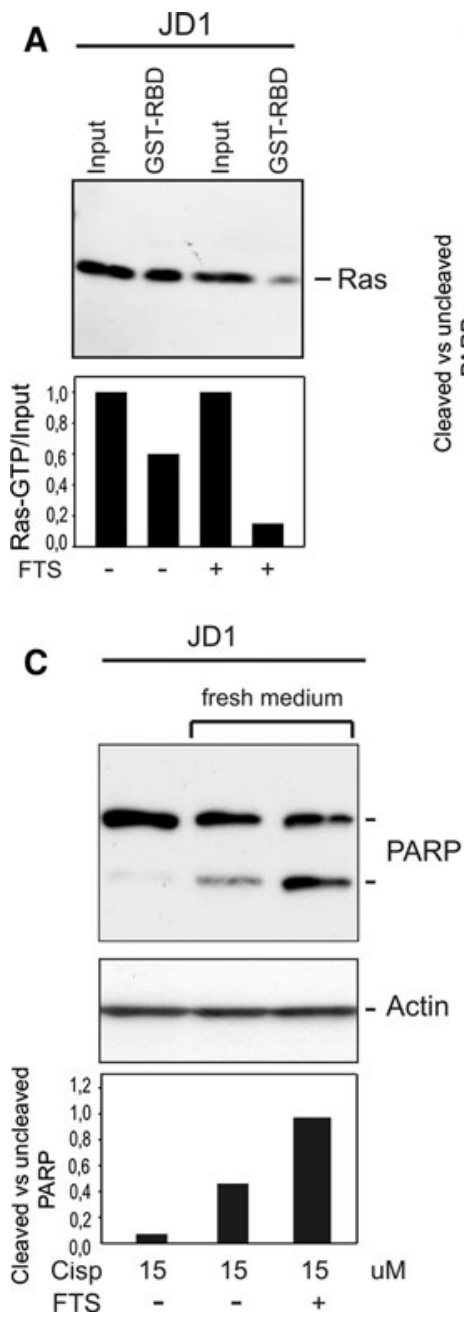

B Jurkat
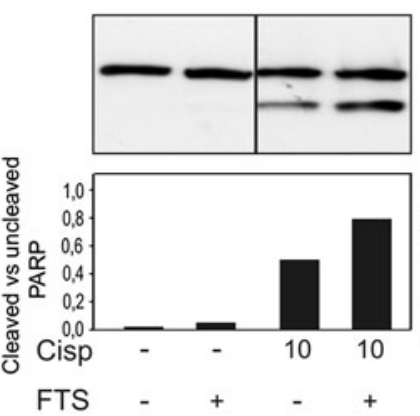

D

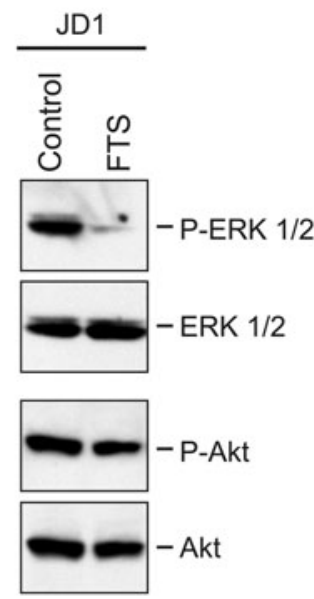

JD1
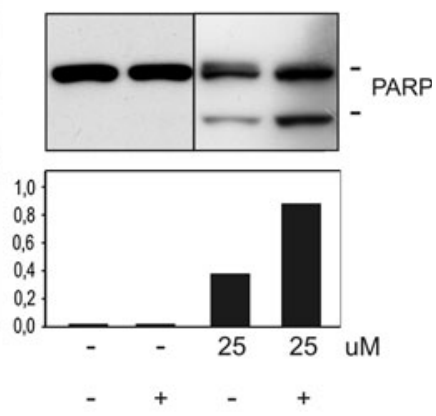

model: we hypothesized an accumulation of the active Ras (i.e., GTP-bound) conformation, due to a defective guanine nucleotide hydrolysis, as a result of Tax/GAP interaction [5].

Here, while investigating the molecular mechanisms of Tax-mediated resistance to apoptosis in T-cells, we found that cells expressing Tax either transiently or constitutively have higher levels of Ras-GTP than their control counterparts. These data are consistent with the proposed mechanism of action of Tax and strengthen previous findings indicating that Tax-mediated resistance to apoptosis is, at least in part, associated with Ras activity. Indeed, we show that inhibition of Ras signaling is accompanied by an increased sensitivity to cisplatin treatment. It was previously reported that a combined treatment with cisplatin and inhibitors of Ras farnesylation increases the cytotoxic effect of the cisplatin alone [45]. In addition, it was also reported that protein geranylgeranylation is essential for ATLL cell survival [46]. Instead of farnesylation or geranylgeranylation inhibitors, we used FTS ( $S$-farnesylthiosalicylic acid), a Ras farnesylcystein mimetic that selectively interacts with the activated form of Ras. FTS has a very low toxicity, and has been reported to restrain the growth of tumor cells in vivo [36, 47, 48]. We found that treatment of JD1 cells with FTS was accompanied by a decrease in Ras-GTP and a parallel increase in sensitivity to cisplatin treatment. Interestingly, increased apoptosis susceptibility of Tax-expressing cells to treatment with FTS was accompanied by a consistent reduction in phospho-ERK, suggesting a direct involvement of ERK activation in the apoptosis protection mediated by Tax. Moreover, although several reports stressed the potential relevance of Akt activation in survival of HTLV-1 infected or Tax-expressing cells, no reduction in phospho-Akt was observed after FTS treatment. The different behavior of ERK and Akt could be the result of the diverse activation pattern of the two proteins. Indeed, ERK activation is directly linked to Ras through the Raf-MEK pathway, whereas Akt is a downstream effector of PI3K, whose activation can be driven not only by Ras but also by diverse inducers [49-51]. Thus, Ras inhibition has likely a more direct and rapid effect in ERK activation. 
Recently, it has been shown that one other protein of HTLV-1, the p13, can act in a opposite manner to Tax favoring cell death by interfering with the Ras pathway [52]; furthermore it has also been reported that nuclear p13 binds Tax and inhibits its transcriptional activity [53]. Thus, analysis of Ras role in lymphocytes expressing the entire HTLV-1 genome will be of great interest.

Nonetheless, our data provide evidence of Ras signaling activation in Tax-expressing T-cells, and indicate this occurrence as a possible cause of ATLL cell resistance to death by chemotherapeutic agents. Although additional studies should evaluate whether the levels of Ras-GTP correlate with disease prognosis and the extent of apoptosis resistance, our data indicate in Ras a possible target for ATLL therapy.

Acknowledgments This study was supported by grants from European Union (Contract No. 2005-018704), the Associazione Italiana per la Ricerca sul Cancro (AIRC), Ministero della Salute (progetto RFPS-2006-2-342010). The authors thank Pierantonio Gallo and Renzo Grancara for artwork.

Conflict of interest The authors declare that they have no competing interest.

Open Access This article is distributed under the terms of the Creative Commons Attribution Noncommercial License which permits any noncommercial use, distribution, and reproduction in any medium, provided the original author(s) and source are credited.

\section{References}

1. Bos JL (1989) Ras oncogenes in human cancer: a review. Cancer Res 49:4682-4689

2. Schubbert S, Shannon K, Bollag G (2007) Hyperactive Ras in developmental disorders and cancer. Nat Rev Cancer 7:295-308

3. McCubrey JA, Steelman LS, Abrams SL et al (2008) Targeting survival cascades induced by activation of Ras/Raf/MEK/ERK, PI3 K/PTEN/Akt/mTOR and Jak/STAT pathways for effective leukemia therapy. Leukemia 22:708-722

4. Scholl C, Gilliland DG, Frohling S (2008) Deregulation of signaling pathways in acute myeloid leukemia. Semin Oncol 35: 336-345

5. Vajente N, Trevisan R, Saggioro D (2009) HTLV-1 Tax protein cooperates with Ras in protecting cells from apoptosis. Apoptosis 14:153-163

6. Hinuma Y, Nagata K, Hanaoka M et al (1981) Adult T-cell leukemia: antigen in an ATL cell line and detection of antibodies to the antigen in human sera. Proc Natl Acad Sci USA 78: 6476-6480

7. Poiesz BJ, Ruscetti FW, Gazdar AF, Bunn PA, Minna JD, Gallo RC (1980) Detection and isolation of type C retrovirus particles from fresh and cultured lymphocytes of a patient with cutaneous T-cell lymphoma. Proc Natl Acad Sci USA 77:7415-7419

8. Gessain A, Barin F, Vernant JC et al (1985) Antibodies to human T-lymphotropic virus type-I in patients with tropical spastic paraparesis. Lancet 2:407-410

9. Grassmann R, Aboud M, Jeang KT (2005) Molecular mechanisms of cellular transformation by HTLV-1 Tax. Oncogene 24:5976-5985
10. Hasegawa H, Sawa H, Lewis MJ et al (2006) Thymus-derived leukemia-lymphoma in mice transgenic for the Tax gene of human T-lymphotropic virus type I. Nat Med 12:466-472

11. Grassmann R, Dengler C, Muller-Fleckenstein I et al (1989) Transformation to continuous growth of primary human $\mathrm{T}$ lymphocytes by human T-cell leukemia virus type I X-region genes transduced by a Herpesvirus saimiri vector. Proc Natl Acad Sci USA 86:3351-3355

12. Robek MD, Ratner L (1999) Immortalization of CD4(+) and CD8(+) T lymphocytes by human T-cell leukemia virus type 1 tax mutants expressed in a functional molecular clone. J Virol 73:4856-4865

13. Boxus M, Twizere JC, Legros S, Dewulf JF, Kettmann R, Willems L (2008) The HTLV-1 tax interactome. Retrovirology 5:76

14. Hanahan D, Weinberg RA (2000) The hallmarks of cancer. Cell 100:57-70

15. Chlichlia K, Khazaie K (2010) HTLV-1 Tax: linking transformation, DNA damage and apoptotic T-cell death. Chem Biol Interact 188:359-365

16. Taylor JM, Nicot C (2008) HTLV-1 and apoptosis: role in cellular transformation and recent advances in therapeutic approaches. Apoptosis 13:733-747

17. Saggioro D, Silic-Benussi M, Biasiotto R, D'Agostino DM, Ciminale V (2009) Control of cell death pathways by HTLV-1 proteins. Front Biosci 14:3338-3351

18. Saggioro D, Barp S, Chieco-Bianchi L (2001) Block of a mitochondrial-mediated apoptotic pathway in Tax-expressing murine fibroblasts. Exp Cell Res 269:245-255

19. Trevisan R, Daprai L, Acquasaliente L, Ciminale V, ChiecoBianchi L, Saggioro D (2004) Relevance of CREB phosphorylation in the anti-apoptotic function of human T-lymphotropic virus type 1 Tax protein in serum-deprived murine fibroblasts. Exp Cell Res 299:57-67

20. Paskalis H, Felber BK, Pavlakis GN (1986) Cis-acting sequences responsible for the transcriptional activation of human T-cell leukemia virus type I constitute a conditional enhancer. Proc Natl Acad Sci USA 83:6558-6562

21. Smith MR, Greene WC (1990) Identification of HTLV-I tax trans-activator mutants exhibiting novel transcriptional phenotypes. Genes Dev 4:1875-1885

22. Hanly SM, Rimsky LT, Malim MH et al (1989) Comparative analysis of the HTLV-I Rex and HIV-1 Rev trans-regulatory proteins and their RNA response elements. Genes Dev 3:15341544

23. Sodroski JG, Rosen CA, Haseltine WA (1984) Trans-acting transcriptional activation of the long terminal repeat of human $\mathrm{T}$ lymphotropic viruses in infected cells. Science 225:381-385

24. Saggioro D, Forino M, Penzo A, Pesce M, Oliviero S, ChiecoBianchi L (1994) Tax-induced HTLV-I LTR transcriptional activation is modulated by phosphorylation. Biochem Biophys Res Commun 205:666-673

25. Chuang SE, Doong SL, Lin MT, Cheng AL (1997) Tax of the human T-lymphotropic virus type I transactivates promoter of the MDR-1 gene. Biochem Biophys Res Commun 238:482-486

26. Lau A, Nightingale S, Taylor GP, Gant TW, Cann AJ (1998) Enhanced MDR1 gene expression in human T-cell leukemia virus-I-infected patients offers new prospects for therapy. Blood 91:2467-2474

27. Krishna R, Mayer LD (2000) Multidrug resistance (MDR) in cancer mechanisms, reversal using modulators of MDR and the role of MDR modulators in influencing the pharmacokinetics of anticancer drugs. Eur J Pharm Sci 11:265-283

28. Bunn PA Jr, Foss FM (1996) T-cell lymphoma cell lines (HUT102 and HUT78) established at the National Cancer Institute: history and importance to understanding the biology, clinical features, and therapy of cutaneous T-cell lymphomas (CTCL) and 
adult T-cell leukemia-lymphomas (ATLL). J Cell Biochem Suppl 24:12-23

29. Nagata K, Ohtani K, Nakamura M, Sugamura K (1989) Activation of endogenous c-fos proto-oncogene expression by human T-cell leukemia virus type I-encoded p40tax protein in the human T-cell line, Jurkat. J Virol 63:3220-3226

30. Trevisan R, Daprai L, Paloschi L, Vajente N, Chieco-Bianchi L, Saggioro D (2006) Antiapoptotic effect of human T-cell leukemia virus type 1 tax protein correlates with its creb transcriptional activity. Exp Cell Res 312:1390-1400

31. Fiskum G, Craig SW, Decker GL, Lehninger AL (1980) The cytoskeleton of digitonin-treated rat hepatocytes. Proc Natl Acad Sci USA 77:3430-3434

32. Herrmann C, Martin GA, Wittinghofer A (1995) Quantitative analysis of the complex between p21ras and the Ras-binding domain of the human Raf-1 protein kinase. J Biol Chem 270:2901-2905

33. Ikezoe T, Nishioka C, Bandobashi K et al (2007) Longitudinal inhibition of PI3 K/Akt/mTOR signaling by LY294002 and rapamycin induces growth arrest of adult T-cell leukemia cells. Leuk Res 31:673-682

34. Jeong SJ, Pise-Masison CA, Radonovich MF, Park HU, Brady JN (2005) Activated AKT regulates NF-kappaB activation, p53 inhibition and cell survival in HTLV-1-transformed cells. Oncogene 24:6719-6728

35. Peloponese JM Jr, Jeang KT (2006) Role for Akt/protein kinase B and activator protein- 1 in cellular proliferation induced by the human T-cell leukemia virus type 1 tax oncoprotein. J Biol Chem 281:8927-8938

36. Rotblat B, Ehrlich M, Haklai R, Kloog Y (2008) The Ras inhibitor farnesylthiosalicylic acid (Salirasib) disrupts the spatiotemporal localization of active Ras: a potential treatment for cancer. Methods Enzymol 439:467-489

37. Cantrell DA (2003) GTPases and T cell activation. Immunol Rev 192:122-130

38. Sabnis AJ, Cheung LS, Dail M et al (2009) Oncogenic Kras initiates leukemia in hematopoietic stem cells. PLoS Biol 7:e59

39. von Lintig FC, Huvar I, Law P, Diccianni MB, Yu AL, Boss GR (2000) Ras activation in normal white blood cells and childhood acute lymphoblastic leukemia. Clin Cancer Res 6:1804-1810

40. Zhang J, Wang J, Liu Y et al (2009) Oncogenic Kras-induced leukemogeneis: hematopoietic stem cells as the initial target and lineage-specific progenitors as the potential targets for final leukemic transformation. Blood 113:1304-1314

41. Delmas C, Heliez C, Cohen-Jonathan E et al (2002) Farnesyltransferase inhibitor, R115777, reverses the resistance of human glioma cell lines to ionizing radiation. Int J Cancer 100:43-48

42. Smalley KS, Eisen TG (2003) Farnesyl transferase inhibitor SCH66336 is cytostatic, pro-apoptotic and enhances chemosensitivity to cisplatin in melanoma cells. Int J Cancer 105:165-175

43. Calvisi DF, Ladu S, Conner EA et al (2011) Inactivation of Ras GTPase-activating proteins promotes unrestrained activity of wild-type Ras in human liver cancer. J Hepatol 54:311-319

44. Wu K, Bottazzi ME, de la Fuente $C$ et al (2004) Protein profile of tax-associated complexes. J Biol Chem 279:495-508

45. Wesierska-Gadek J, Kramer MP, Schmid G (2008) A combined treatment of HeLa cells with the farnesyl protein transferase inhibitor L-744, 832 and cisplatin significantly increases the therapeutic effect as compared to cisplatin monotherapy. J Cell Biochem 104:189-201

46. Nakada M, Uota S, Saitoh Y et al (2009) Role for protein geranylgeranylation in adult T-cell leukemia cell survival. Exp Cell Res 315:141-150

47. Kloog Y, Cox AD (2000) RAS inhibitors: potential for cancer therapeutics. Mol Med Today 6:398-402

48. Haklai R, Elad-Sfadia G, Egozi Y, Kloog Y (2008) Orally administered FTS (salirasib) inhibits human pancreatic tumor growth in nude mice. Cancer Chemother Pharmacol 61:89-96

49. Cleary JM, Shapiro GI (2010) Development of phosphoinositide3 kinase pathway inhibitors for advanced cancer. Curr Oncol Rep 12:87-94

50. Knowles MA, Platt FM, Ross RL, Hurst CD (2009) Phosphatidylinositol 3-kinase (PI3 K) pathway activation in bladder cancer. Cancer Metastasis Rev 28:305-316

51. Bunney TD, Katan M (2010) Phosphoinositide signalling in cancer: beyond PI3 K and PTEN. Nat Rev Cancer 10:342-352

52. Hiraragi H, Michael B, Nair A, Silic-Benussi M, Ciminale V, Lairmore M (2005) Human T-lymphotropic virus type 1 mitochondrion-localizing protein p13II sensitizes Jurkat $\mathrm{T}$ cells to Ras-mediated apoptosis. J Virol 79:9449-9457

53. Andresen V, Pise-Masison CA, Sinha-Datta U et al (2011) Suppression of HTLV-1 replication by Tax-mediated rerouting of the p13 viral protein to nuclear speckles. Blood 118:1549-1559 